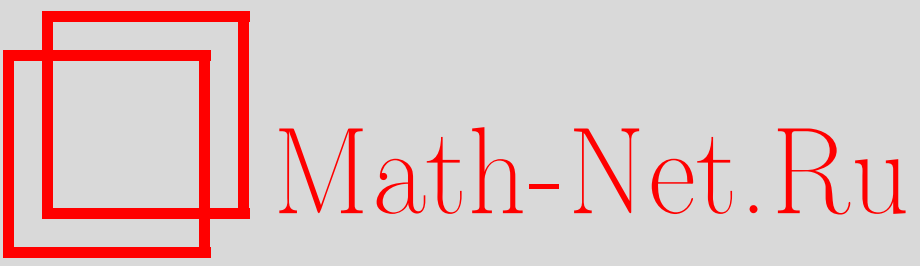

О. В. Бесов, В. И. Ильин, Л. Д. Кудрявцев, В. П. Курдюмов, С. М. Никольский, Л. В. Овсянников, В. А. Садовничий, Станислав Иванович Похожаев (к шестидесятилетию со дня рождения), УМH, 1996, том 51, выпуск 2, 183-188

DOI: https://doi.org/10.4213/rm1569

Использование Общероссийского математического портала Math-Net.Ru подразумевает, что вы прочитали и согласны с пользовательским соглашением http://www.mathnet.ru/rus/agreement

Параметры загрузки:

IP: 35.173 .137 .237

26 апреля 2023 г., $12: 22: 41$ 


\section{СТАНИСЛАВ ИВАНОВИЧ ПОХОЖАЕВ \\ (к шестидесятилетию со дня рождения)}

24 августа 1995 года исполнилось 60 лет известному математику, члену-корреспонденту Российской академии наук Станиславу Ивановичу Похожаеву, крупному специалисту в области нелинейного анализа.

Станислав Иванович родился в семье служащих в Забайкальском селе Усть-Кяхта. Его отец Иван Стефанович, инженер-гидротехник, работал в это время на реке Селенга.

В 1952 г. после окончания средней школы в г. Краснодаре он поступил в Московский физико-технический институт на аэромеханический факультет. Окончив институт в 1958 г., С.И. Похожаев поступил в аспирантуру М $\Phi$ ТИ по специальности математика и занимался в ней под руководством Л.В. Овсянникова. В связи с организацией Сибирского отделения АН СССР Л.В. Овсянников переехал в Новосибирск, а вместе с ним и С.И. Похожаев.

В 1961 г. Станислав Иванович защитил кандидатскую диссертацию "Исследование краевой задачи для уравнения $\Delta u=u^{2} "$

В 1963 г. С.И. Похожаев вернулся в Москву, где работал последовательно: доцентом, профессором, зав. кафедрой высшей математики в Московском энергетическом институте.

В 1970 г. Станислав Иванович защитил докторскую диссертацию "О некоторых нелинейных уравнениях".

В 1984 г. Станислав Иванович был избран членом-корреспондентом АН СССР по отделению математики.

С 1986 г. С.И. Похожаев работает главным научным сотрудником в Математическом институте им. В.А. Стеклова в отделе теории функций.

Первые исследовательские работы Станислав Иванович выполнил еще студентом. Его студенческая работа по сверхзвуковым газовым струям, выполненная под руководством Г. Г. Черного, была опубликована в 1958 г.

В его дипломной работе, посвященной сверхзвуковым турбулентным струям, был предложен оригинальный подход, связываюший непосредственно профиль распределения температур с профилем скоростей. Это позволило установить факт перераспределения температур торможения в свободных сверхзвуковых турбулентных струях.

В работах 1960-1961 г.г. для уравнения $\Delta u=u^{2}$ были впервые получены теоремы существования двух решений, а также отсутствия вешественного решения задачи Дирихле в зависимости 
от граничных данных. Здесь же впервые было установлено явление разрушения ("blow-up") peшения на всей границе области.

В 1964 г. С.И. Похожаев обращается к проблеме существования собственных функций для квазилинейных эллиптических краевых задач. С этой целью он получает и публикует в 1965 г. теорему о непрерывном и компактном вложении пространств Соболева $W_{p}^{l}(\Omega), \Omega \in \mathbb{R}^{N}$, в случае $p l=N$, в соответствующие пространства Орлича. Здесь же строится контрпример, показьвающий неулучшаемость полученных резултатов.

По-видимому, наибольшую известность Станиславу Ивановичу принесло открытие им принципиально новых энергетических тождеств для квазилинейных эллиптических уравнений второго порядка (1965) и произвольного порядка с нелинейной частью дивергентного вида (1970). Эти тождества получили название тождеств Похожаева. Они позволили впервые получить теоремы отсутствия собственных функций для квазилинейных эллиптических краевых задач. В результате ему удалось создать общую и законченную теорию собственных функций нелинейных эллиптических задач. Полученные им здесь условия являются неулучшаемыми. Эти результаты, полученные для квазилинейных эллиптических задач произвольного порядка, явились новыми (и неожиданными) даже для квазилинейных уравнений второго порядка.

Характерной чертой исследований Станислава Ивановича является подход к конкретнњм нелинейным задачам с общих позиций нелинейного функционального анализа. Так в связи с проблемой собственных функций Станислав Иванович получает (1968) теорему о множестве критических значений фредгольмовых функционалов. В связи с тем, что конечномерная теория Морса-Сарда не переносится на функционалы в бесконечномерном случае (даже для функционалов класса $C^{\infty}$ на гильбертовом пространстве), Станислав Иванович уточняет классическую теорему А. Морса и вводит понятие фредгольмового функционала. Именно к этому классу принадлежат функционалы, порождаемые вариационными квазилинейными эллиптическими задачами при естественных условиях в соответствующих пространствах Соболева.

После создания теории нелинейных монотонных коэрцитивных операторов (М.И. Вишик, F. Browder и др.) возникла проблема разрешимости нелинейных уравнений с некоэрцитивными операторами.

В 1967 г. Станислав Иванович определяет и выделяет класс усиленно-замкнутых нелинейных операторов (впоследствии названных $A$-собственными операторами), для которых были впервые установлены теоремы разрешимости без условия коэрцитивности. Для этих операторов (нечетных и однородных в главном) впервые в нелинейном анализе была установлена нелинейная альтернатива Фредгольма.

Развивая теорию разрешимости нелинейных уравнений, Станислав Иванович в 1969-1970 гг. вводит понятие нормалњной разрешимости нелинейных уравнений, обобщающее известное понятие нормальной разрешимости по Хаусдорфу линейных уравнений. В это же время дается приложение к нелинейным уравнениям с частными производными. В частности, эта общая теория нормальной разрешимости дала возможность установить глобальную разрешимость общих нелинейных параболических задач при условии существования априорных оценок, к которым не применим метод Лере-Шаудера.

Интересы Станислава Ивановича не ограничиваются эллиптическими задачами.

В 1971 г. Станислав Иванович получает теоремы существования периодических решений для квазилинейных гиперболических однородных уравнений в многомерном случае.

В 1975 г. он устанавливает глобальную разрешимость смешанных задач для одного общего класса нелинейных (в главном) гиперболических уравнений, названных им "уравнениями Кирхгофа". Впоследствии Станислав Иванович открыл в этом классе уравнений Кирхгофа "странные нелинейные многомерные" гиперболические уравнения, обладающие "странными свойствами". Этот класс уравнений был не известен ранее даже в одномерном случае.

В 1980 г. Станислав Иванович ставит и успешно решает проблему создания общей теории априорных оценок для слабо нелинейных эллиптических уравнений. С помощью разработанного им метода интерполяции и вложения нелинейных операторов он установил точные характеристики роста нелинейных операторов, необходимые для разрешимости соответствующих задач. Эти результаты позволили установить и понять внутреннюю природу и происхождение ряда ограничений на рост нелинейных операторов. В частности, оказалось, что известное условие Бернштейна-Нагумо является лишь предельным случаем условия разрешимости уравнений вида $\Delta u=f(x, u, \nabla u)$ в пространстве $W_{p}^{2}(\Omega)$ при $p \rightarrow \infty$. На основании развитой им теории были получены теоремы разрешимости для широкого класса квазилинейных эллиптических уравнений произвольного порядка недивергентной структуры.

При исследовании проблемы существования гладких априорных оценок впервые была выяв- 
лена роль критического показателя нелинейности в некоэрцитивных эл липтических задачах. Было установлено существование порога гладкости, не зависящего ни от гладкости нелинейности, ни от гладкости данных задачи даже для эллиптических уравнений второго порядка с главным оператором Лапласа.

Болшшой цикл работ С. И. Похожаева посвящен глобальному изучению решений нелинейных операторных уравнений, в частности, нелинейных эллиптических задач. Им изучены операторы, не обладающие свойством коэрцитивности, что представляет большой интерес не только с точки зрения общей теории, но и потому, что такая ситуация часто встречается в важных с точки зрения приложений задачах. Здесь С.И. Похожаев также создал принципиально новый метод - метод сферического расслоения и разработал ряд его обобщений. Применение этого метода позволило исследовать новые классы нелинейных задач, в том числе и задачи с четной нелинейностью.

Особо следует отметить, что метод сферического расслоения С.И. Похожаева позволяет установить как разрешимость, так и отсутствие решений у соответствующих задач. При этом в некоторых случаях также появляется возможность доказать существование кратных решений и даже существование счетного множества решений.

В 1990 г. Станис лав Иванович обращается к проблеме существования целых решений для квазилинейных эллиптических уравнений. Он предлагает новый подход к изучению этих проблем и получает ряд принципиально новых результатов в этой области. При этом были доказаны теоремы существования с точными первыми членами асимптотики решений для нового широкого класса квазилинейных эллиптических уравнений. Им был изучен случай не только докритического роста нелинейной части уравнения, но и впервые суперкритический случай ее роста, где им впервые было установлено существование континуума решения во всем пространстве $\mathbb{R}^{N} \mathrm{c}$ фиксированной скоростью убывания на бесконечности для нелинейных уравнений с ненулевой правой частью. Для уравнений нелинейных скалярных полей с произвольным радиальным возмущением установлены точные асимптотические оценки их поведения на бесконечности. Эти оценки, не улучшаемые как относительно характера их нелинейности, так и относительно возмущения, получены практически во всем диапазоне степенных нелинейностей.

В последние годы С.И. Похожаев установил глобальные теоремы существования для модифицированных уравнений Томаса-Ферми и совместно с голландским математиком L. Peletier - для расширенного уравнения Колмогорова-Петровского-Пискунова.

Результаты Станислава Ивановича многократно докладывались на Всесоюзных конференциях, а также на Международных конференциях, проходивших как в нашей стране, так и за рубежом: Польша (1991, 1994), Франция (1991), Япония (1992), Швейцария (1993, 1994), Германия (1992, 1995), Нидерланды (1994, 1995), Бельгия (1994), Чехия (1995), Италия (1995), Испания (1995).

Наряду с научной деятельностью С.И. Похожаев ведет интенсивную педагогическую и научно-организационную работу. С 1971 по 1986 годы он заведовал кафедрой высшей математики в Московском энергетическом институте и содействовал там существенному улучшению педагогического процесса. Он читал и продолжает читать лекции по различным математическим дисциплинам. Его лекции, предельно четкие и ясные, заслуженно высоко ценятся студентами и преподавателями. С 1976 г. он является членом президиума Научно-методического совета по математике сначала при Минвузе СССР, а ныне при Госкомитете по высшему образованию РФ. На этой должности он активно содействует поднятию уровня математического образования в технических высших учебных заведениях, повышению его роли и значения. Много сил, и не безуспешно, им было потрачено на то, чтобы кафедры математики в вузах заняли более высокое, чем это было раньше, положение, достойное их действительной значимости. С 1992 г. С.И. Похожаев является заместителем главного редактора журнала "Дифференциальные уравнения" и членом редколлегии журнала "Математические заметки".

Много лет С.И. Похожаев руководит научно-исследовательским семинаром по нелинейному анализу, где выросло немало его учеников, докторов и кандидатов наук. С.И. Похожаев щедро делится своим опытом, знаниями и идеями со своими учениками и умело направляет их работу на решение принципиальных и актуальных задач, своевременно приходя на помощь .

За свою плодотворную работу Станислав Иванович награжден орденом Дружбы народов и многими Почетными грамотами.

Желаем Станиславу Ивановичу Похожаеву оставаться таким же бодрым и жизнерадостным, каким он всегда является, и ждем от него новых интересных научных результатов.

О. В. Бесов, В.И. Ильин, Л. Д. Кудрявиев, С. П. Курдюмов, С. М. Никольский, Л. В. Овсянников, В.А. Садовничий 


\section{СПИСОК НАУЧНЫХ ТРУДОВ С. И. ПОХОЖАЕВА}

[1] Об одном вопросе сверхзвукового истечения // Труды МФТИ. 1958. №1. С. 167-172

[2] О задаче Дирихле для уравнения $\Delta u=u^{2} / /$ ДАН СССР. 1960. Т. 134. № 3. С. 769-772

[3] Аналог метода Шмидта для нелинейного уравнения. Труды МФТИ // ДАН СССР. 1960. Т. 136. № 3. С. 546-548

[4] О краевой задаче для уравнения $\Delta u=u^{2} / /$ ДАН СССР. 1961. Т. 138. № 2. С. 305-308

[5] Исследование краевой задачи для уравнения $\Delta u=u^{2} / /$ Дисс. . . . канд. физ.-матем. наук. Новосибирск, 1961

[6] Постановка задачи о сильном взрыве на поверхности жидкости // ДАН СССР. 1962. Т. 144. № 3. С. 524-526 (совм. с Дерибасом А.А.)

[7] О теореме вложения С.Л. Соболева в случае $p l=n / /$ Доклады научно-технической конференции МЭИ, секция матем. М., 1965. С. 158-170

[8] О собственных функциях уравнения $\Delta u+\lambda f(u)=0 / /$ ДАН СССР. 1965. Т. 165. № 1 . C. $36-39$

[9] Об одном классе операторов и разрешимости квазилинейных эллиптических уравнений // Матем. сб. 1967. Т. 72. № 2. С. 226-236 (совм. с Дубинским Ю.А.)

[10] О разрешимости нелинейных уравнений с нечетными операторами // Функц. анализ и его прилож. 1967. Т. 1. №3. С. 66-73

[11] О собственных функциях некоторых нелинейных задач // Доклады научно-технической конференции МЭИ, секция матем. М., 1967. С. 186-191

[12] О множестве критических значений функционалов // Матем. сб. 1968. Т. 75 . №1. C. $106-111$

[13] О нормальной разрешимости нелинейных уравнений // ДАН СССР. 1969. Т. 184. №1. C. $40-43$

[14] О нелинейных операторах, имеющих слабо замкнутую область значений, и квазилинейных эллиптических уравнениях // Матем. сб. 1969. Т. 78. № 2. С. 237-259

[15] Нормальная разрешимость нелинейных уравнений в равномерно выпуклых банаховых пространствах // Функц. анализ и его прилож. 1969. Т. 3. № 2. С. 90-94

[16] О множествах уровня функционалов // Доклады научно-технической конференции МЭИ, секция матем. М., 1969. С. 63-68

[17] Законы сохранения и априорные оценки для некоторых параболических уравнений // Дифференц. уравнения. 1970. Т. 6. №1. С. 129-136

[18] О собственных функциях квазилинейных эллиптических задач // Матем. сб. 1970. Т. 82. № 2. C. $192-212$

[19] О слабо нелинейных гиперболических системах // Труды ММО. 1970. Т. 23. С. 161-176

[20] К разрешимости квазилинейных гиперболических систем // ДАН СССР. 1970. Т. 192. №6. С. $1217-1220$

[21] О некоторых нелинейных уравнениях // Дисс. . . докт. физ.-матем. наук. М., 1970. C. 350

[22] Об одном квазилинейном параболическом уравнении // Дифференц. уравнения. 1971. T. 7. № 1. C. $73-80$

[23] О периодических решениях некоторых нелинейных гиперболических уравнений // ДАН CCCP. 1971. T. 198. №6. С. $1274-1277$

[24] О некоторых некоэрцитивных квазилинейных эллиптических задачах // Труды МЭИ. Математика. 1971. № 89. С. 11-19

[25] Об одном классе квазилинейных гиперболических систем // Труды МЭИ. Математика. 1972. C. $11-19$

[26] Квазилинейные гиперболические уравнения Кирхгофа и законы сохранения // Труды МЭИ. Исследования по дифференциальным уравнениям и их приложениям. 1974. № 201. C. $11-19$

[27] Об одном классе квазилинейных гиперболических уравнений // Матем. сб. 1975. Т. 96. № 1. С. $152-166$

[28] Исследование гиперболических систем квазилинейных уравнений методом продолжения // Труды МЭИ. 1975. №260. С. 74-88

[29] Вопросы отсутствия решения нелинейных краевых задач // Труды Всесоюзной конференции по уравнениям с частными производными. М.: Изд-во МГУ, 1978. С. 200-203

[30] Об уравнениях неизоэнтропического движения газов // Труды МЭИ. Прикладные вопросы математики. 1978. № 357. С. 81-87 
[31] Об уравнениях вида $\Delta u=f(x, u)$ (тезисы доклада) // УМН. 1978. Т. 33. № 3. С. 149-149

[32] Об одном подходе к нелинейным уравнениям // ДАН СССР. 1979. Т. 247. №6. C. $1327-1331$

[33] Теорема сравнения и отсутствие решений нелинейных краевых задач // Труды МЭИ. Прикладные задачи математики. 1980. № 499. С. 42-48

[34] О периодических решениях некоторых нелинейных систем обыкновенных дифференциальных уравнений // Дифференц. уравнения. 1980. Т. 16. №1. С. 109-116

[35] Об уравнениях вида $\Delta u=f(x, u) / /$ Матем. сб. 1980. Т. 113. № 2. С. 324-338

[36] О новой программе по математике для инженерно-технических специальностей ВУЗов // Сборник научно-методических статей по математике. №9, 1981. С. 5-12 (совм. с Никольским С.М., Кудрявцевым Л. Д., Кузнецовым Л.А.)

[37] О региональных совещаниях-семинарах заведующих математическими кафедрами // Сборник научно-методических статей по математике. №9, 1981. С. 113-122 (совм. с Кудрявцевым Л. Д., Кузнецовым Л.А.)

[38] О квазилинейных эллиптических уравнениях высокого порядка // Дифференц. уравнения. 1981. T. 17. № 1. С. $115-128$

[39] О подчиненных операторах нелинейных уравнений // ДАН СССР. 1981. Т. 257. №6. C. $282-286$

[40] Нелинейные уравнения с частными производными // Математическая энциклопедия. T. 3, 1982. C. $950-956$

[41] О разрешимости квазилинецных эллиптических уравнений произвольного порядка // Матем. сб. 1982. Т. 117. № 2. С. 251-265

[42] К общей теории априорных оценок решений нелинейных уравнений // Труды МЭИ. Прикладные задачи математики. 1982. №566. С. 62-69

[43] О разрешимости квазилинейных эллиптических уравнений высокого порядка // Дифференц. уравнения. 1982. Т. 18. №1. С. 100-109

[44] $\mathrm{K}$ общей теории априорных оценок решений квазилинейных уравнений // Сборник докладов 7 Советско-Чехословацкого семинара по задачам математической физики. Ереван, 1982. С. 280-285

[45] Об одном подходе к нелинейным уравнениям и его приложениях // УМН. 1982. Т. 37. № 2. C. 261

[46] К общей теории априорных оценок решений нелинейных уравнений (тезисы доклада) // УМН. 1982. Т. 37. № 4. С. 90

[47] Вложение нелинейных операторов и априорные оценки решений нелинейных уравнений // ДАН СССР. 1982. Т. 266. № 5. С. 1063-1066

[48] Об априорных оценках решений нелинейных уравнений // Тезисы докладов Всесоюзной конференции: Лаврентьевские чтения. Новосибирск, 1982. С. 24-25

[49] Об априорных оценках решений квазилинейных эллиптических уравнений произвольного порядка // Дифференц. уравнения. 1983. Т. 19. № 1. С. 101-110

[50] Об одном методе расслоения в нелинейных задачах // Межведомственный сборник по математике. М.: МЭИ, 1984. С. 7-12

[51] Об одном квазилинейном гиперболическом уравнении Кирхгофа // Дифференц. уравнения. 1985. Т. 21. № 1. С. 101-108

[52] Редактор перевода: Фарлоу С. Уравнения с частными производными. М.: Мир, 1985

[53] О вложении нелинейных операторов и априорных оценках решений нелинейных уравнений // Дифференциальные уравнения в частных производных и их приложения. Труды Всесоюзного симпозиума, 1982. Тбилиси, 1986. С. 203-210

[54] Редактор перевода: Куфнер А., Фучик С. Нелинейные дифференциалњные уравнения. М.: Наука, 1986

[55] Об одном классе классе нелинейных некоэрцитивных задач // Исследования по теории операторов. Уфа: Уральское отделение АН СССР. Башкирский научный центр. Институт математики, 1988. С. 5-17

[56] Об одном конструктивном методе вариационного исчисления // ДАН СССР. 1988. T. 298. № 6. С. $1330-1333$

[57] Об одной задаче Л.В. Овсянникова // ЖПМТиТФ 1989. № 2. С. 5-10

[58] Нелинейный прикладной функциональный анализ. М.: Изд-во МЭИ, 1989 (совм. с Жариновым В.В., Илларионовым М.А., Пикулиным В.П.)

[59] Практический курс по уравнениям математической физики. М.: Изд-во МЭИ, 1989 (совм. с Пикулиным В.П.) 
[60] О методе расслоения решения нелинейных краевых задач // Труды МИАН. 1990. C. $146-163$

[61] О разрешимости эллиптической задачи в $\mathbb{R}^{N}$ с суперкритическим показателем нелинейности // ДАН СССР. 1990. Т. 313. №6. С. 1356-1360

[62] О классах положительности эллиптических операторов в $\mathbb{R}^{N}$ с суперкритическим показателем нелинейности // ДАН СССР. 1990. Т. 314. №3. С. 558-561

[63] Об одном новом интегральном соотношении и его приложениях к нелинейным эллиптическим задачам // III Международная конференция: Лаврентьевские чтения по математике, механике и физике, 10-14 сентября 1990 г. Тезисы докладов, 1990. С. 34

[64] Об эллиптических задачах в $\mathbb{R}^{N}$ с суперкритическим показателем нелинейности // Матем. сб. 1991. Т. 182. №4. С. 467-489

[65] О целых решениях квазилинейных эллиптических уравнений // ДАН СССР. 1991. T. 318. № 4. C. $815-820$

[66] О целых решениях одного класса квазилинейных эллиптических уравнений // ДАН CCCP. 1991. T. 318. № 6. C. 1319-1324

[67] Об асимптотике целых радиальных решений квазилинейных эллиптических уравнений // ДАН СССР. 1991. Т. 320. № 4. С. 808-813

[68] On entire solutions of semilinear elliptic equations. Progress in Partial Differential Equations: Elliptic and Parabolic Problems // Proc. of I-st European Conference on Elliptic-Parabolic Problems. V. 266: Pitman Research Notes in Mathematics, 1991 (C. Bandle at al (ed.)) ; New York: Longman Scientific Technical. P. 56-69

[69] О гладкости решений некоторых суперлинейных эллиптических уравнений // ДАН. 1992. T. 327. № 3. C. 299-302

[70] О точных априорных оценках для некоторых суперлинейных эллиптических уравнений // ДАН. 1992. Т. 327. № 4-6. С. 433-437

[71] О квазилинейных эллиптических задачах в $\mathbb{R}^{N}$ в суперкритическом случае // Труды МИАН. 1992. Т. 201. С. $324-341$

[72] О целых радиальных решениях некоторых квазилинейных эллиптических уравнений // Матем. сб. 1992. Т. 183. №11. С. 3-18

[73] Об одной математической модели электролиза // ДАН. 1993. Т. 332. №6. С. 18-20

[74] О целых радиальных решениях квазилинейных эллиптических уравнений // Труды МИАН. 1993. Т. 204. С. 251-273

[75] Точные априорные оценки для одной квазилинейной вырождающейся эллиптической задачи // Матем. сб. 1993. Т. 184. № 8. С. 3-16

[76] Точные априорные оценки для некоторых эллиптических уравнений // Дифференц. уравнения. 1993. Т. 29. № 3. С. 472-486

[77] The Sharp Apriori Estimates for Some Superlinear Degenerate Elliptic Problems // Function Spaces, Differential Operators and Nonlinear Analysis. V. 133. Leipzig: Teubner-Stuttgart, 1993. P. 200-217

[78] Об одной нелинейной задаче электролиза // Матем. сб. 1994. Т. 185. №5. С. 103-118

[79] Об одной нелинейной задаче Г. Аманна // Дифференц. уравнения. 1994. Т. 30. № 4. C. $675-691$

[80] Об одной квазилинейной системе уравнений электрохимической диффузии // Труды МИРАН. 1995. Т. 210. С. 239-263

[81] Об уравнениях Маслова // Дифференц. уравнения. 1995. Т. 31. № 2. С. 338-349

[82] Практический курс по уравнениям математической физики. М.: Наука, 1995 (совм. с Пикулиным В.П.)

[83] The Cauchy problem for the extended Ficher-Kolmogorov (EFK) equation (to appear) (with L.A. Peletier)

[84] Global positive solutions for a fourth order parabolic equation (to appear) (with L.A. Peletier)

[85] Positive solutions for the $p$-Laplacian: Application of the fibering method (to appear) (with P. Drabek)

О. В. Бесов, В. И. Ильин, Л. Д. Кудрявцев, С. П. Курдюмов, С. М. Никольский, Л.В. Овсянников, В.А. Садовничий 